\title{
Heavy-Ion Irradiation of Thulium(III) Oxide Targets Prepared by Polymer-Assisted Deposition
}

Mitch A. Garcia ${ }^{\text {a,b }}$, Mazhar N. Ali ${ }^{\text {a,b }}$, Noel N. Chang ${ }^{\text {a,b }}$, Tashi Parsons-Moss ${ }^{\text {a,b }}$, Paul D. Ashby ${ }^{\text {b }}$, Jacklyn M. Gates ${ }^{\text {a,b }}$, Liv Stavsetra ${ }^{\text {b }}$, Kenneth E. Gregorich ${ }^{\text {b }}$, Heino Nitsche ${ }^{\text {a,b }}$

${ }^{a}$ Department of Chemistry, University of California Berkeley, Berkeley, California 94720, USA

${ }^{b}$ Lawrence Berkeley National Laboratory, Berkeley, California 94720, USA

\begin{abstract}
Thulium(III) oxide $\left(\mathrm{Tm}_{2} \mathrm{O}_{3}\right)$ targets prepared by the polymer-assisted deposition (PAD) method were irradiated by heavy-ion beams to test the method's feasibility for nuclear science applications. Targets were prepared on silicon nitride backings (thickness of $1000 \mathrm{~nm}, 344 \mu \mathrm{g} / \mathrm{cm}^{2}$ ) and were irradiated with an ${ }^{40} \mathrm{Ar}$ beam at laboratory frame energy of $\sim 210 \mathrm{MeV}$ (50 particle $\mathrm{nA}$ ). The root mean squared (RMS) roughness prior to irradiation is $1.1 \mathrm{~nm}$ for a $250 \mathrm{~nm}\left(\sim 220 \mu \mathrm{g} / \mathrm{cm}^{2}\right) \mathrm{Tm}_{2} \mathrm{O}_{3}$ target, and an RMS roughness of $2.0 \mathrm{~nm}$ after irradiation was measured by atomic force microscopy (AFM). Scanning electron microscopy of the irradiated target reveals no significant differences in surface homogeneity when compared to imaging prior to irradiation. Target flaking was not observed from monitoring Rutherford scattered particles as a function of time.
\end{abstract}

This work was supported by the Director, Office of Science, of the U.S. Department of Energy under Contract No. DE-AC02-05CH11231. 


\section{Paper}

Targets are an essential component in experimental nuclear science as a source of stationary nuclei for nuclear reactions with heavy-ion beams. Typically, targets should be chemically-pure, uniform, homogeneous, and crack-free over the irradiated area, while also being structurally rigid. Conventional methods of preparing targets include: cold rolling [1], vacuum evaporation [2], molecular plating [3], painting/sedimentation [4], electrodeposition [5], and die compaction [6]. Each method has advantages and disadvantages with regards to achievable target thicknesses, homogeneity, chemical forms, reproducibility, and deposition yields. Recently, Jia et al. developed polymerassisted deposition (PAD) as an alternative method to prepare thin films of metal oxides [7]. We have shown previously that the PAD method yields highly uniform metal oxide films that may be suitable as targets for nuclear science [8]. Herein, we report the results from heavy-ion irradiation of several metal oxide targets, prepared by the PAD method, at the 88-Inch Cyclotron of the Lawrence Berkeley National Laboratory (LBNL). Characterizations of the targets after irradiation were performed by scanning electron microscopy (SEM) and atomic force microscopy (AFM). Target integrity during irradiation was monitored by Rutherford scattering as a function of time.

The metal oxide targets were prepared as follows. A low-pressure chemical vapor deposition (LPCVD) furnace (tystar, TYTAN) was used to epitaxially grow low-stress silicon nitride $\left(\mathrm{Si}_{3} \mathrm{~N}_{4}\right)$ onto both sides of 4-in single-sided polished (SSP) silicon wafers [100]. Dichlorosilane and ammonia with flow rates of 100 and 25 sccm, respectively, were used as the silicon nitrating source at $18.7 \mathrm{~Pa}$ and $835{ }^{\circ} \mathrm{C}$. The film stress was about $200 \mathrm{MPa}$ and the deposition rate was $40 \AA / \mathrm{min}$. The $\mathrm{Si}_{3} \mathrm{~N}_{4}$ film was produced from a 
standard protocol calibrated to yield $1000 \pm 20 \mathrm{~nm}\left(344 \pm 7 \mu \mathrm{g} / \mathrm{cm}^{2}\right)$ before etching. Four rectangles $(1.8 \times 1.3 \mathrm{~cm})$ were scratched through the $\mathrm{Si}_{3} \mathrm{~N}_{4}$ layer, on the unpolished (bottom) side of the silicon wafers, to expose the silicon surface. These patterned wafers were then placed for $8-10 \mathrm{~h}$ in a $20 \%$ potassium hydroxide bath at $80{ }^{\circ} \mathrm{C}$, to etch away the silicon and leave only a rectangular surface of $\mathrm{Si}_{3} \mathrm{~N}_{4}$.

A solution of $8 \%$ by weight (b.w.) thulium with $15 \%$ (b.w.) polyethylenimine (PEI) in water was prepared exactly as previously described by Garcia et al. [8]. A 3-mL aliquot of the thulium solution was spread on top of wafers containing the four $\mathrm{Si}_{3} \mathrm{~N}_{4}$ windows. The wafers were spun at $1500 \mathrm{rpm}$ and then baked over a period of $3 \mathrm{~h}$ in a muffle oven to a final temperature of $600{ }^{\circ} \mathrm{C}$. The process creates a thin layer of thulium(III) oxide $\left(\mathrm{Tm}_{2} \mathrm{O}_{3}\right)$, while the polymer decomposes to volatile oxides. A second layer of $\mathrm{Tm}_{2} \mathrm{O}_{3}$ was coated on the wafer by adding an additional $300 \mathrm{~mL}$-aliquot of the 8\% (b.w.) thulium solution and repeating the steps described above. The final thickness of the $\mathrm{Tm}_{2} \mathrm{O}_{3}$ film was $\sim 250 \mathrm{~nm}\left(\sim 220 \mu \mathrm{g} / \mathrm{cm}^{2}\right)$ as measured from taking the average of several cross-section images by SEM. The wafers were scribed and broken into pieces of $7.6 \times 6.0 \mathrm{~cm}$. The outer silicon was used as a frame to support the metal oxide film and silicon nitride window on a target-ladder for heavy-ion irradiation.

Two $\operatorname{Tm}_{2} \mathrm{O}_{3}$ targets were each irradiated separately with a different heavy-ion beam of either $208 \mathrm{MeV}$ Argon $\left({ }^{40} \mathrm{Ar}^{10+}\right)$, or $180 \mathrm{MeV}$ Argon $\left({ }^{40} \mathrm{Ar}^{8+}\right)$. The first target was irradiated with the $\mathrm{Ar}^{10+}$ beam for $35 \mathrm{~min}$ at a beam current of $3.1 \times 10^{11}$ particles/s (50 particle $\mathrm{nA}$ ) for a total dose of $6.6 \times 10^{14}$ particles. A second target was irradiated with the $\mathrm{Ar}^{8+}$ beam for $17 \mathrm{~min}$ at a maximum beam current of $1.2 \times 10^{12}$ particles/s 
(200 particle $\mathrm{nA}$ ) for a total dose of $1.3 \times 10^{15}$ particles. The irradiated targets were easily removed from the target ladder and were subject to further analysis.

Visual inspection of the $\mathrm{Tm}_{2} \mathrm{O}_{3}$ target irradiated by the ${ }^{40} \mathrm{Ar}^{10+}$ beam revealed a darkened rectangular region, see supplementary materials. The dimensions and location of the darkened rectangle are consistent with the known beam spot image after beam collimation. The $\mathrm{Tm}_{2} \mathrm{O}_{3}$ target irradiated by the higher intensity (200 particle $\left.\mathrm{nA}\right){ }^{40} \mathrm{Ar}^{8+}$ beam, had a melt spot after irradiation. This was done to test the limits of the method as stationary targets only receive a maximum intensity of $\sim 40$ particle nA during an irradiation to prevent melting. The melt spot for the intensely irradiated $\mathrm{Tm}_{2} \mathrm{O}_{3}$ target indicates an upper-limit of beam intensity that stationary targets prepared by the PAD method can withstand. From monitoring the quantity of Rutherford scattered particles as a function of time, no decrease in intensity for either target was observed; this indicates target flaking did not occur during the irradiation.

An SEM (Ultra 55VP Fesem, Zeiss) with an acceleration voltage of $5 \mathrm{kV}$ was used to determine surface homogeneity of the ${ }^{40} \mathrm{Ar}^{10+}$ irradiated target at a magnification of 274x. A cross-sectional SEM image was also taken to determine uniformity at a magnification of $31 \mathrm{kx}$. A representative image of the surface after the irradiation is shown in figure 1a. Small surface aggregates randomly distributed on the surface can be seen, and were present before irradiation. This is likely due to using non-clean room techniques in the application of the PAD method. A small pinhole $\sim 7 \mathrm{x} 4 \mu \mathrm{m}$ is also visible in the upper right hand side of figure 1a. Pinholes are seen prior to irradiation and are likely due to defects in the substrate. A representative SEM cross-section of the target after irradiation is shown in figure $1 \mathrm{~b}$; two layers of $\operatorname{Tm}_{2} \mathrm{O}_{3}$ can be clearly seen in the 
figure. The back edge was tilted $\sim 8^{\circ}$ toward the top of the wafer, in order to image the surface of $\mathrm{Tm}_{2} \mathrm{O}_{3}$ in tandem with viewing the striated $\mathrm{Tm}_{2} \mathrm{O}_{3}$ layers. The figure shows the high degree of uniformity achieved with the PAD method. It should be noted that previous experiments utilizing the reapplication of the PAD method did not show layer striations [8]. The occurrence of striation is likely dependent on the annealing temperature profile. The SEM analyses of the irradiated target show no significant deviation from those imaged prior to irradiation.

An AFM (MFP 3D, Asylum Research) was used to obtain a high resolution image of a $1 \times 1 \mu \mathrm{m}^{2}$ representative section of the irradiated target surface and a non-irradiated portion of the same target for the ${ }^{40} \mathrm{Ar}^{10+}$ irradiation. The AFM used cantilevers with a spring constant of $3 \mathrm{~N} / \mathrm{m}$ (Multi75, Budget Sensors) for imaging in the attractive regime in AC mode. The AFM images are shown in figure 2a-b. The homogeneity and granular appearance of the $\mathrm{Tm}_{2} \mathrm{O}_{3}$ layer on the non-irradiated portion of the target is consistent with previous experiments with a root mean square (RMS) roughness of $1.1 \mathrm{~nm}$. The irradiated portion, figure $2 \mathrm{~b}$, also shows a homogeneous surface with low surface variability with an RMS roughness of $2.0 \mathrm{~nm}$. After irradiation, the homogeneity of the film is slightly reduced.

From monitoring the Rutherford scattered particles during irradiation it was observed that there was no loss of target material. This is in contrast to other methods which may produce targets that flake even before irradiation [9]. Loss of target material through the course of a beam irradiation will decrease the overall production of the desired nuclear reaction channel, and may prelude the undesired consequence of contamination if the target is composed of a radioactive isotope. Another consequence of 
target flaking is the loss of expensive enriched isotopic material. Therefore, the PAD method is an advantageous target methodology for preparing uniform, homogeneous crack-free metal oxides for heavy-ion irradiation. Further experiments are planned to determine target temperature during irradiation and the optimum thickness of silicon nitride to minimize energy-transfer from heavy-ions, while retaining high structural integrity.

This work was supported by the National Nuclear Security Administration under the Stewardship Sciences Academic Alliance Program, Project NS00075, Award Number DE-FG52-06NA27480. Work at the Molecular Foundry was supported by the Director, Office of Science, Office of Basic Energy Sciences, Division of Materials Sciences and Engineering, of the U.S. Department of Energy under Contract No. DE-AC0205CH11231 


\section{Figures}

Figure 1. (a) Representative surface SEM of the irradiated $\mathrm{Tm}_{2} \mathrm{O}_{3}$ film produced from the PAD method using an $8 \%$ by weight Tm solution. (b) Representative cross-section SEM of the same $\mathrm{Tm}_{2} \mathrm{O}_{3}$ film.

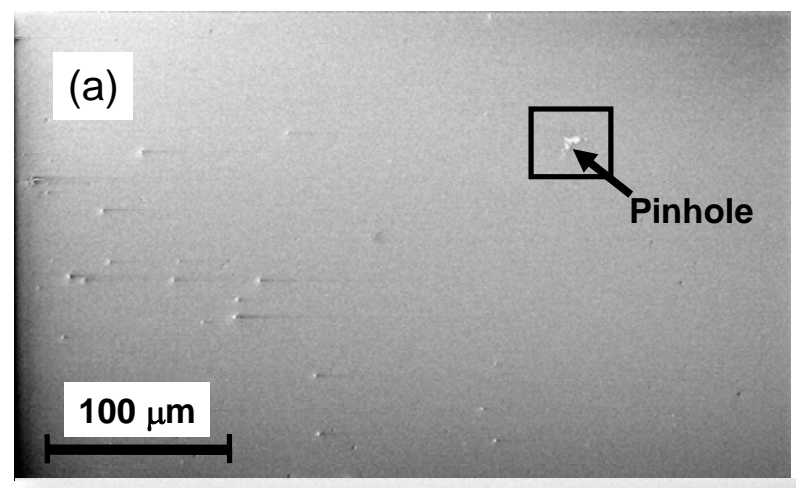

(b)
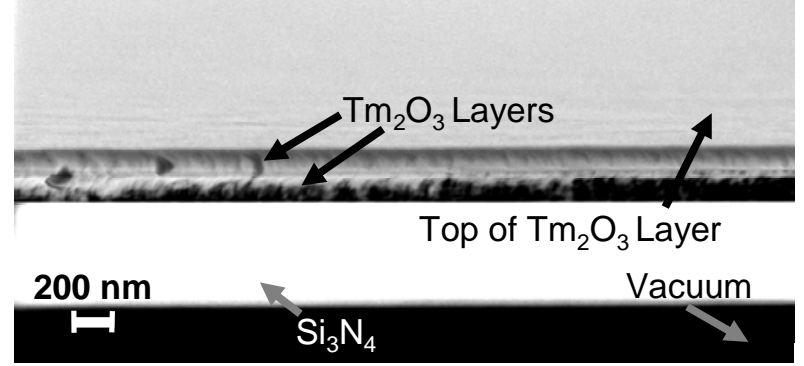
Figure 2. (a) Representative AFM of the non-irradiated area of $\mathrm{Tm}_{2} \mathrm{O}_{3}$ film produced from the PAD method (b) Representative AFM of the irradiated area of $\mathrm{Tm}_{2} \mathrm{O}_{3}$ film produced by the PAD method.

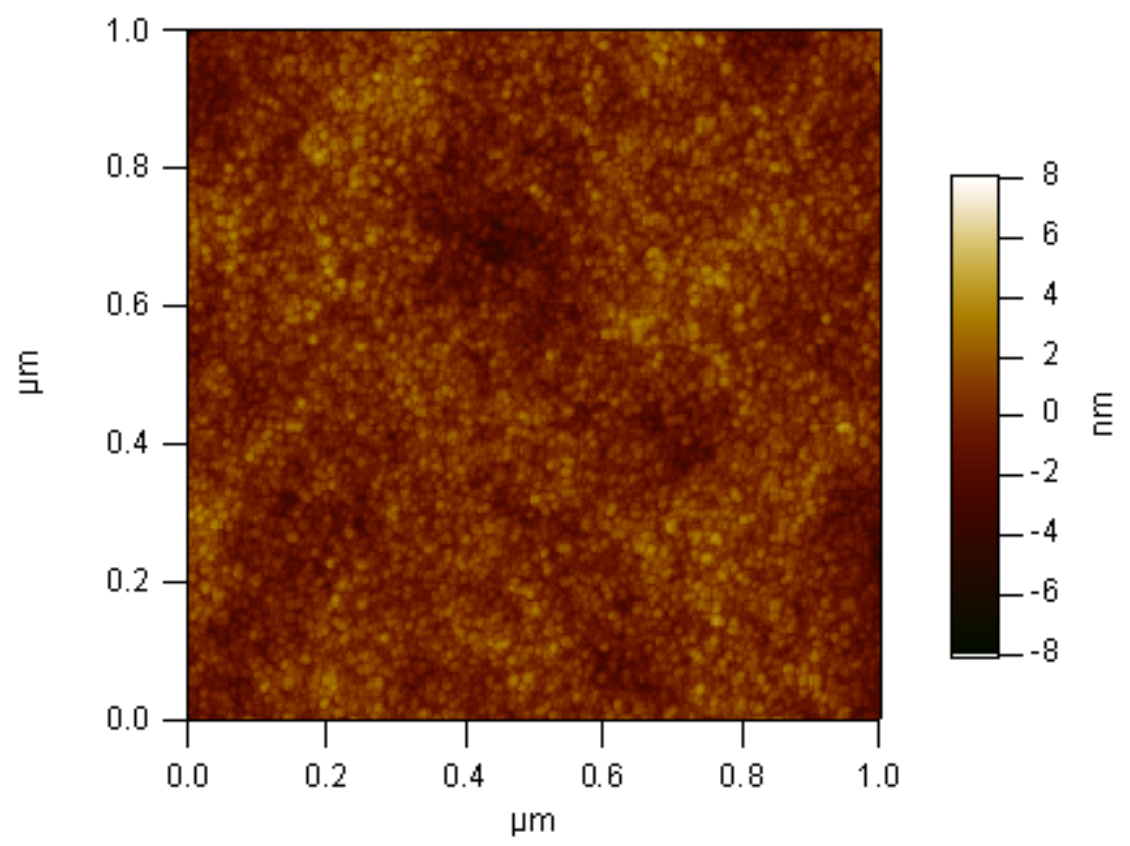

\section{References}

[1] S. Clifford, X. Guo-ji, C. Ingelbrecht, M.J. Pomeroy, Nucl. Instrum. Meth. A 480 (2002) 29.

[2] R. Grossmann, H.J. Maier, H.U. Friebel, D. Frischke, Nucl. Instrum. Meth. A 480 (2002) 209.

[3] K. Eberhardt, M. Schädel, E. Schimpf, P. Thörle, N. Trautmann, Nucl. Instrum. Meth. A 521 (2004) 208.

[4] L.V. Drapchinsky, T.E. Kuzmina, S.M. Soloviev, Nucl. Instrum. Meth. A 438 (1999) 116.

[5] S. Chakrabarty, B.S. Tomar, A. Goswami, V.A. Raman, S.B. Manohar, Instrum. Meth. B 174 (2001) 212.

[6] K.M. Glover, F.J.G. Rogers, T.A. Tuplin, Nucl. Instrum. Methods 102 (1972) 443.

[7] Q.X. Jia, M. McCleskey, A.K. Burrell, Y. Lin, G.E. Collis, H. Wang, A.D.Q. Li, S.R. Foltyn, Nat. Mater. 3 (2004) 529.

[8] doi:10.1016/j.tsf.2007.11.127

[9] W.S. Aaron, M. Petek, L.A. Zevenbergen, J.R. Gibson, Nucl. Instrum. Meth. A 282 (1989) 147. 